\title{
Don't hesitate, innovate
}

Looking at your research with a business mentality can be surprisingly satisfying, says Ying Lia Li.

$\mathrm{T}$ he Nature/Entrepreneur First Innovation Forum was launched this year with the aim of nurturing five teams of physicists interested in starting a quantum technology company. An intensive 6-week training period provided by Entrepreneur First, a company specializing in accelerating deep-technology start-ups, culminated in a day of presentations, on 6 May, to a panel of scientific experts, entrepreneurs and industry leaders. As one of the chosen participants who presented, I'm sharing my insight into the first steps of spinning out research.

It may seem premature to start a quantum tech company. However, early adopters (defence, space, and research and development (R\&D) companies), financial investors (angel investors, and established deep-technology companies), and governments are actively searching for the next disruptive technology. The UK government is committed to spending $£ 270$ million on the National Quantum Technologies programme, while the 2016-2017 Innovate UK budget for emerging

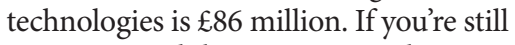
not convinced that quantum tech is worth investing in, look at D-Wave, who has secured a total investment worth US\$174 million.

I applied for the Innovation Forum on the basis of my PhD project with Peter Barker, which successfully demonstrated cooling the motion of microspheres using optical whispering-gallery-mode resonances. These resonances form when trapped laser light bounces around the sphere billions of times due to total internal reflection, enhancing detection of the sphere's motion relative to a coupling waveguide. My ambitious business idea builds on this displacement detection to form a quantum-limited optical accelerometer where the microsphere is cooled to its centre-of-mass quantum ground state. Such an exquisitely sensitive device could potentially measure accelerations less than $10^{-10} \mathrm{~m} \mathrm{~s}^{-2}$; resolving gravitational field gradients in space, detecting underground oil reserves or allowing for indoor navigation without the use of GPS.

I soon realized that this initial business concept was extremely naive. Academics are accustomed to pushing fundamental concepts such as quantum sensing to their absolute limits. However, such precision or hardware complexity is often beyond

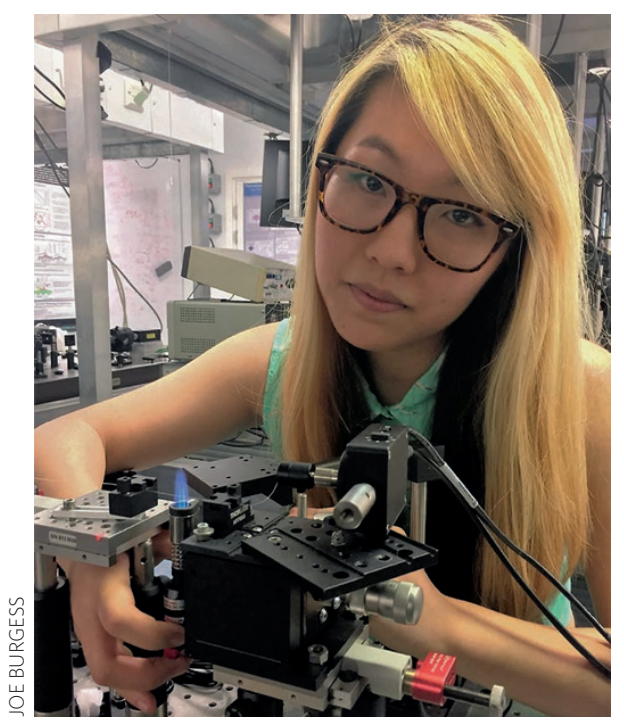

real-world applications. Even if industry wanted such sensitivity, developing a quantum-limited accelerometer would be risky considering that other academic devices such as atom interferometers can outperform our device already. The only way to compete is to consider the situation from a market point of view.

An experimental project can involve multiple grants, the sweat and tears of many $\mathrm{PhD}$ students, and an extensive list of bespoke equipment. However, industry investors want a solid core team and a device they can see, touch and, most importantly, test. Based on these points, we stripped down the development of the device to a manageable workload for a team of two, and evaluated the level of quantum features versus the precision, cost and competitive edge. An accelerometer using classical physics capable of resolving $10^{-7} \mathrm{~m} \mathrm{~s}^{-2}$ could be miniaturized, prototyped and tested in the field within a typical business cycle of 5 years; much faster and cheaper than any of our competitors. As Andrew Heller (IBM) once said, "Technology is like fish, the longer it stays on the shelf the less desirable it becomes." This became the cornerstone of my pitch - a competitively priced classical sensor with a compelling plan to develop a product line of quantumenabled sensors with market longevity.

Each time I spoke to a potential customer, competitor or mentor, my strategy became more mission driven. I learnt from past disruptive technologies, such as seeking collaborative R\&D with industry, similar to the development of optical fibres, and avoiding the high initial costs of microelectromechanical systems foundries. I no longer tried to promote my research but, instead, worked to win customers by listening to their needs. I trained myself to not accept simple responses such as 'we don't need quantum-limited sensing, or even worse 'it sounds interesting' (followed by static silence). Instead, I'd ask what issues they have with their current sensors, 'it's too heavy', 'we can't calibrate it ourselves. There's a name (and a free book) for this method called The Mom Test.

The Innovation Forum presentations provided some valuable take-home messages, for example, the overhyping of patents. The panel pointed out that filing patents too early sets a profit-making deadline and may have consequences, with both university spin-outs and customers citing unpleasant experiences when handling intellectual property tied to institutions. Another piece of advice is understanding technology maturity to identify areas that require further incubation. For example, those working on quantum computing protocols or quantum networking devices should ask themselves what the total hardware cost would be and whether these devices can first be integrated into existing platforms instead of into a newly designed quantum computer. The key is to not let the future of your company hinge on someone else developing the infrastructure. Adapt for the market now, create success stories, but keep championing the benefits of further quantum capabilities.

When my presentation ended I knew it was just the beginning of my journey. Any initial hesitation based on feeling inadequately business savvy or needing more academic publications had all dissipated. Starting a company is exciting, it builds leadership skills and distinguishes you as a trailblazer where you are respected and praised, despite the naysayers. So why not try it? In the words of Entrepreneur First, 'the corporates will still want you if you fail.'

YING LIA LI is a PhD student in the Department of Physics and Astronomy, University College London, London WC13 6BT, UK. 